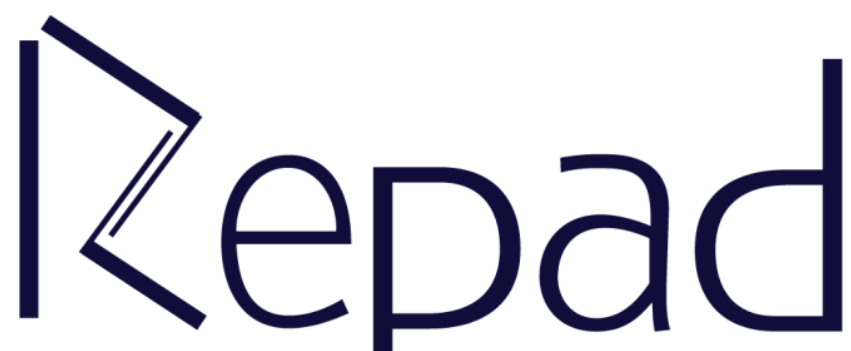

Vol. 3, n. 1, Abril/2019

Revista Estudos e

Pesquisas em Administração

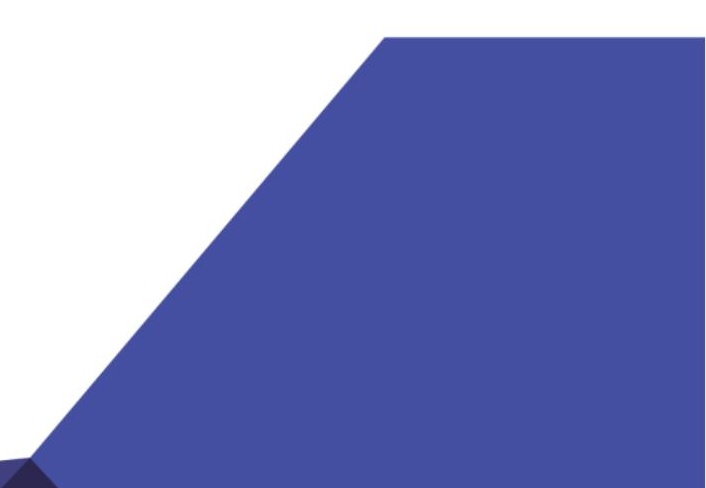




\title{
PANORAMA DOS ESTUDOS DE CASO DISPONÍVEIS NA PLATAFORMA DA SCIENTIFIC PERIODICALS ELETRONIC LIBRARY - SPELL
}

\author{
Hipólito Mendes \\ Universidade Federal de Mato Grosso \\ https://orcid.org/0000-0002-2680-3763 \\ André Roese \\ Universidade Federal de Mato Grosso \\ https://orcid.org/0000-0001-6722-0428
}

\begin{abstract}
RESUMO
Apesar das críticas do passado por alguns cientistas sobre o questionamento de credibilidade do método de Estudo Caso, atualmente, dentre os métodos de pesquisa, o Estudo de Caso é um dos mais populares. Em alguns momentos as pesquisas que utilizaram esse método foram alvos de críticas no meio científico e em alguns casos confundido como um simples método ou estágio de pesquisa de caráter exploratório, ou como uma pesquisa etnográfica ou de observação participante. Portanto, há uma necessidade de imergir no campo de Estudo de Caso para melhor compreender como e quando se aplica. Uma das formas para esse entendimento é via análise das publicações científicas nos periódicos dependendo de período do interesse do pesquisador. Assim, este artigo objetivou analisar os estudos de caso publicados na base SPELL na área temática de gestão ambiental, nos anos 2015 a 2018. A análise se desdobrou em categorias como: nome das revistas publicadas, quantidades de artigos, principais palavras chave e classificação Qualis/CAPES por revista. O método utilizado para o levantamento dos dados desta pesquisa foi a partir da base científica Spell (Scientific Periodicals Electronic Library), utilizando-se "Gestão Ambiental" como palavra-chave. Quanto ao método de seleção de artigos, foram escolhidos os classificados como Estudo de Caso, publicados em resumos e periódicos entre 2015-2018, nas seguintes áreas de conhecimento: Administração, Economia, Engenharia, no idioma em Português. Quanto aos resultados apresentados na amostra dos vinte e um (21) artigos analisados, oito (8) artigos foram publicados na Revista de Gestão Ambiental e Sustentabilidade, três (3) artigos na Revista Desenvolvimento em Questão, dois (2) na Revista de Administração da Universidade Federal de Santa Maria - UFSM, dois (2) artigos na Revista Gestão e Sociedade, e um artigo em cada uma das outras cinco revistas encontradas.
\end{abstract}

Palavras-chave: Estudo de Caso. Gestão Ambiental. Spell

\section{LANDSCAPE OF CASE STUDIES AVAILABLE ON THE SCIENTIFIC PERIODICALS ELETRONIC LIBRARY - SPELL}

\begin{abstract}
Despite some scientists' criticisms of the past on the question of the Case Study method credibility, currently, among the research methods, case study is one of the most popular. In some moments, studies using this method have been targets of criticism in the scientific world. In addition, the case study has been misunderstood as a simple method, an exploratory research stage, and even mistaken for the ethnographic research or participant observation. Therefore, there is a need to immerse in the field of case study to better understand how and when it applies. One way for it is via analysis of the scientific publications in journals depending on the period of interest of the researcher. Thus, this article aimed to analyze case studies published on the
\end{abstract}


base SPELL in the thematic area of environmental management, from 2015 to 2018. The analysis involved categories such as: names of the journals published, number of articles, main keywords and Qualis/CAPES rate by journal. The method used for data collection was the scientific base Spell (Scientific Periodicals Electronic Library). We used "environmental management" as the keyword. As the method for article selection, we chose the ones classified as case study, published in the Portuguese language in the following areas of knowledge: Business Administration, economics and engineering. As for the approach, this work is of quantitative/qualitative nature. Twenty-one (21) articles were analyzed and the results showed that: eight (8) were published in the "Gestão Ambiental e Sustentabilidade" journal, three (3) in the "Desenvolvimento em Questão" journal, two (2) in the Administration Journal of the Federal University of Santa Maria - UFSM, two (2) in the "Gestão e Sociedade" journal and one article for each of the five journals found.

Keywords: Case Study. Environmental management. Spell

\section{CONSIDERAÇÕES INICIAIS}

A pesquisa científica tende a ser estruturada em forma de construção arquitetada, em que o pesquisador precisa antes de tudo, entender como percorrer pelos caminhos para chegar os objetivos almejados. Desta forma, os pesquisadores são desafiados em cumprirem certas regras paradigmáticas que possa os levar ao destino almejado. Conforme Kuhn (2006), qualquer pessoa que deseja descrever e analisar a evolução de método científico primeiramente teria que aceitar as regras e paradigmas do método.

A partir do momento que o pesquisador aceitar os desafios paradigmáticos, portanto, terá que escolher a abordagem metodológica de análise de resultado: qualitativa ou quantitativa, contudo as duas abordagens têm diferentes áreas específicas de aplicação, mas não descarta a possibilidade de aplica-los simultaneamente. No estudo de caso, por se tratar de estudo que preocupa compreender e descrever os fenômenos tende a utilizar a abordagem qualitativa sem ignorar por completo a quantitativa, como afirmam Meirinhos e Osório (2010), que o estudo de caso aceita dicotomia qualitativa e quantitativa de tratamento dos dados baseados em interpretação construtivista.

Desse modo, o estudo de caso procura analisar os fenômenos contemporâneos da vida real de (os) sujeito (os), grupos sociais, evento entre outros, Freitas e Jabbour (2011), afirmam que o estudo de caso é um procedimento metodológico que enfatiza a percepção contextual, que leva em consideração as representatividades, concentrando-se num estudo profundo e exaustivo de um ou mais objetos, de forma que permita a sua explicação mais detalhada. Hoje, o estudo de caso tem sido utilizado em publicações em diferentes áreas do conhecimento inclusive da gestão ambiental, administração de empresas, enfermagem, educação, psicologia entre outras.

Este artigo tem como objetivo, analisar as publicações dos artigos sobre estudo de caso na área temática de gestão ambiental na base da SPELL, nos anos 2015 a 2018. Nosso principal objetivo é descrever os nomes das revistas publicadas, quantidades de artigos publicados, principais palavras chave e classificação QUALIS/ CAPES por revista. Desta maneira, surge um questionamento? Em que medida o estudo de caso está sendo utilizado por diferentes pesquisadores em gestão ambiental e como pode contribuir na publicação do conhecimento científico. 


\section{REFERENCIAL TEÓRICO}

O pesquisador antes de iniciar a pesquisa, a primeira coisa a ser feita é a definição do objetivo e qual tipo de abordagem a ser aplicada ao tema: qualitativo, quantitativo, ou uma combinação entre ambas. Para isso, Freitas e Jabbour (2011) classificam as pesquisas sociais em três grupos: Estudos exploratórios, estudos descritivos e estudos que verificam hipóteses causais, também denominada pesquisa explicativa.

Os autores definem que os estudos exploratórios são aqueles que procuram disseminar as ideias e soluções na tentativa de adquirir maior familiaridade com o fenômeno em estudo; a descritiva por sua vez expõe caraterísticas de um grupo da população e o fenômeno entre variáveis para determinar a sua origem. O estudo Qualitativo (YIN, 2010; FREITAS; JABBOUR, 2011; GODOY, 1995), mostram que, o pesquisador não preocupa em quantificar ou enumerar os eventos estudados, não utiliza as ferramentas matemáticas/estatística, na análise dos resultados obtidos na pesquisa, o mesmo, preocupa-se em obtenção e descrição de dados como pessoas, lugares e processos que envolvem a interação de um fenômeno.

Na pesquisa qualitativa, a fonte de coleta dos dados é o ambiente, e esse ambiente não necessita de métodos estatísticos para coletar as informações, ele baseia-se na descrição do fenômeno. $\mathrm{O}$ foco é na abordagem e na interpretação do fenômeno e não no resultado. Enquanto que a abordagem quantitativa permite o pesquisador mensurar os eventos opiniões, hábitos, atitudes, reações etc. através de uso de método estatístico (FREITAS; JABBOUR, 2011). Tendo feita a definição de finalidade da pesquisa, torna-se importante o pesquisador decidir que tipo de abordagem convém para chegar aos objetivos da sua investigação.

Nesse sentido, é necessário que o pesquisador saiba quando aplicar cada uma das abordagens, considerando o que Freitas e Jabbour (2001, p. 9) indicam sobre "quando a finalidade da investigação é descritiva ou causal, a abordagem é quantitativa; quando a finalidade é explicar ou descrever um evento ou uma situação, a abordagem adotada deve ser a qualitativa". Contudo, há a possibilidade de o pesquisador utilizar as duas abordagens (qualitativa e quantitativa) quando achar necessário. Por exemplo, uma pesquisa pode iniciar com abordagem qualitativa e terminar com análise de evidência das informações obtidas por meio de abordagem quantitativa e vice-versa.

Este tipo de pesquisa que combina os dois métodos é conhecido de "triangulação metodológica" ou mixed methodolgy. Essa combinação metodológica é considerada no mundo acadêmico como pesquisa robusta, uma vez que ultrapassa os limites de duas abordagens.

Para Godoy (1995a), o uso de abordagem qualitativa e quantitativa se define como esforço cuidadoso na averiguação e obtenção de novas informações e, consequentemente, na ampliação do conhecimento existente. A autora salienta que, em linhas gerais, o pesquisador que opta na abordagem qualitativa tende a definir a priori o plano bem estabelecido que o possa conduzir de melhor maneira para alcançar seus objetivos e operacionalizá-los com clareza.

Godoy (1995b, p. 21) admite a que existe alguns atributos que definem a pesquisa qualitativa, segunda a autora, "um fenômeno pode ser mais bem compreendido no contexto em que ocorre e do qual é parte, devendo ser analisada numa perspectiva integrada", nessa perspectiva o pesquisador ao realizar a investigação no campo, procura captar o fenômeno de acordo com a percepção das pessoas envolvidas e considerar todas as opiniões válidas para sua análise.

Conforme Freitas e Jabbour (2011), a abordagem qualitativa é um método complexo que exige muito do pesquisador, e pode ser aplicada em situações complexas, de natureza socialmente difícil de mensurar. A dificuldade que se apresenta neste tipo de estudo, refere-se a métodos utilizados para análise que não são delineados em termos estatísticos como são os de 
abordagem quantitativa. Os autores afirmam que os pesquisadores enfrentam grandes dificuldades ao realizarem a pesquisa qualitativa em ordem:

a) Da coleta e análise dos dados de pesquisa qualitativa ser trabalhosa;

b) Da tendência de sobrecarregar o pesquisador em vários pontos, como a exigência de considerável de dispêndio de tempo para organizar e transcrever as gravações, quando for o caso;

c) $\mathrm{Na}$ ausência de clareza dos métodos de análise, diferentemente das análises quantitativas que apresentam convenções claras para pesquisador utilizar.

A pesquisa qualitativa apresenta grandes vantagens em relação à quantitativa pelo fato de sua abrangência e a profundidade nas evidências das informações por meio de múltiplas fontes, por exemplo, entrevistas, observações, análise de documentos, que facilita o pesquisar informações relevantes que tornaria difícil de alcançar na abordagem quantitativa.

\section{ESTUDO DE CASO COMO MÉTODO DE PESQUISA QUALITATIVA}

De acordo com Severino (2007), a ciência dispõe do seu método próprio que utiliza e que o uso desse método, torna o elemento principal do processo de organização do conhecimento alcançado no mundo da ciência, tanto para diferencia-lo das outras modalidades de conhecimento fundamentado em expressão da subjetividade humana, como a religião, a arte, e a filosofia quanto, do senso comum.

O estudo de caso é um procedimento metodológico que enfatiza a percepção contextual, levando em consideração a representatividades, concentrando-se num estudo profundo e exaustivo de um ou mais objetos, de forma que permita a sua explicação mais detalhada (FREITAS; JABBOUR, 2011). Versa-se de um conjunto de procedimento lógicos e técnicas que permitem a operacionalização e relação entre causas e fenômenos.

Nesse pressuposto, a atividade inicial do pesquisador é saber realizar observação dos fatos que a priori nos parece causal e espontânea, daí que entra o papel do pesquisar utilizando a sua capacidade lógica para entender a causa do fenômeno (SEVERINO, 2007, p. 103). Para Andrade et al. (2017), a pesquisa de estudo de caso exige do pesquisador a cautela no momento de preparar o protocolo, onde deve explicar determinados procedimentos formais e ter consciência dos pontos fortes e possíveis limitações no fenômeno em estudo.

Conforme Yin (2009), o estudo de caso pode ser entendido como uma investigação empírica que investiga um fenômeno contemporâneo em profundidade e em seu contexto de vida real, sobretudo quando os limites entre o fenômeno não são claramente evidenciados.

O autor salienta que a investigação de estudo de caso depara com a situação tecnicamente diferenciada nas quais existirão mais variáveis de interesse que pontos de dados. Para obtenção de resultados com múltiplas fontes de evidência, o pesquisador deve buscar evidências para convergir às descobertas durante a triangulação.

Para Severino (2007), o estudo de caso a concentra no caso único, e deve reunir os fatos considerados representativos de um conjunto de casos análogos. Isto é, o caso escolhido para estudo de caso deve ter uma significativa representativa com vista a permitir a generalização em situações análogas.

Em rigor, a coleta dos dados deve respeitar os procedimentos da pesquisa do campo, pois conforme consideram Freitas e Jabbour (2011), o estudo de caso é uma história de acontecimentos do passado ou atual, construídos a partir de múltiplas fontes de evidências, que pode abarcar os procedimentos como observação, entrevistas sistemáticas, como também incluir a pesquisa em documentos. 
Para Andrade et al. (2017), o estudo de caso é um método de pesquisa estruturado, cujo a sua aplicação pode ser em diferentes circunstâncias para contribuir no campo de conhecimento quer nos fenômenos individuais assim como em grupos e que, o estudo de caso possui as suas particularidades e caraterísticas próprias. Para Godoy (1995b), o mesmo é caraterizado por um estudo que se interessa em entender uma unidade em profundidade, ou seja, visa aplicar a investigação detalhada de um fenômeno simples ou particular.

Coraiola et al. (2014) desconsideram o método de estudo de caso como método de pesquisa, mas é uma seleção do que o pesquisador pretender investigar, ou seja, casos individuais. O estudo de caso é uma unidade especifica com um sistema delimitado cujas suas partes estão limitadas. Para Yin (2009), a pesquisa de estudo de caso pode classificada como caso único, assim como, múltiplos.

Em outras circunstâncias, o estudo de caso pode incluir alguns detalhes até mesmo serem limitadas a evidência de abordagem qualitativa. Entretanto, qualquer evidência tanto qualitativa, quanto quantitativa, não distinguem os vários métodos de pesquisa.

O método de estudo de caso não é apenas uma forma de pesquisa qualitativa uma vez que, em algumas pesquisas de estudo de caso vai além do limite de estudo qualitativo, usando a mistura de evidências qualitativas e quantitativas. Além do mais, a pesquisa de estudo de caso não necessita frequentemente incluir a evidência da observação direta e detalhada como tem sido verificado em outras formas de pesquisas qualitativas (YIN, 2009).

\section{TIPOS DE ESTUDOS DE CASO}

Embora tenha sido citado caso de decisões como centro de atenção de estudo do caso. Porém, para uma definição de estudo de caso como método de estudo é insignificante. Podemos, entretanto, encontrar ou incluir alguns casos comuns como "indivíduos, organizações, processos, programas, vizinhanças, instituições assim como eventos" (YIN, 2010, p. 38), dessa forma, citar apenas um tópico de estudo não justifica estabelecer a definição justa e adequada de método de estudo de caso (YIN, 2010).

$\mathrm{O}$ autor aponta que, o estudo de caso foi censurado no passado, por exemplo, nos "antigos livros-textos de ciências sociais" ao considerar que o estudo de caso é um estágio exploratório para as pesquisas de outras áreas de conhecimento. Outro erro comum apontado pelo autor seria confundir o estudo de caso com os estudos etnográfico ou observação participativa.

No tópico anterior foram apresentadas, a partir da obra de Yin (2009), as possíveis definições de estudo do caso. Entretanto, isso demonstra que o método de estudo de caso não se aplica apenas a uma técnica de coleta dos dados de maneira discreta, mas sim, compreende uma abordagem abrangente. $\mathrm{O}$ estudo de caso reporta quatro tipos de aplicações como ilustra (YIN, 2010, p. 41):

- O mais importante é explorar os presumidos vínculos causais nas intervenções na vida real que são demasiado complexos para as estratégias de levantamento ou experimento;

- Uma segunda aplicação é descrever uma intervenção e o contexto da vida real no qual ele ocorreu;

- Em terceiro lugar, os estudos de caso podem ilustrar determinados tópicos em uma avaliação, novamente em modo descritivo; e

- Em quarto lugar, a estratégias de estudo de caso pode ser usada para explorar as situações em que a intervenção sendo avaliada não possui um único e claro conjunto de resultados.

Nesse pressuposto, o estudo de caso pode ser explorado e conduzido de diferentes formas e motivações, que variam de caso para caso, desde os mais individuais até os mais complexos. 


\section{PREPARAÇÃO, HABILIDADES E PROTOCOLO DE ESTUDO DO CASO}

A preparação antecede a execução de qualquer projeto de vida individual ou socialmente comunitário. O estudo de caso, por ser um método científico que procura descrever fenômenos sociais, não foge desse procedimento formal. Aliás, como estuda fenômenos contemporâneos da vida real dos sujeitos humanos, portanto, o pesquisador deve em primeiro planeja como desenvolver as atividades, além de proteger ao máximo a identidade dos sujeitos participantes da pesquisa. Essa necessidade se dá com o envolvimento direto dos sujeitos na pesquisa, ao contrário de pesquisas de sistemas naturais em que o pesquisador fica isolado no campo fazendo seu trabalho sem precisar da intervenção direta dos sujeitos humanos (YIN, 2010).

Segundo Yin (2010), a proteção dos sujeitos é de inteira responsabilidade do pesquisador, isto é, cuidar e conduzir o estudo com sensibilidade. Esse cuidado e sensibilidade dependem e variam de acordo com cada estudo de caso a ser desenvolvido.

Em alguns casos, o pesquisador é obrigado a seguir as orientações da entidade a que pertence como associação de pesquisadores ou conselhos de pesquisas nas Universidades.

No geral, o cuidado envolve: a) obter o consentimento informado de possíveis sujeitos que irão compor a equipe de estudo de caso, onde será detalhado o motivo de estudo e que a participação deve ser de espontânea liberdade; b) proteger os participantes no estudo de caso no máximo possível a eventual "dano" que pode acontecer; c) assegurar a privacidade e a confiabilidade dos participantes para que não fiquem constrangidos e que tenham vontade em participar em outros estudos posteriores; e d) ter cuidado especial para proteger os grupos vulneráveis, isto é, quando o estudo envolve as crianças.

O trabalho de qualquer natureza exige do profissional certas qualidades que lhes permitirá executar as tarefas com eficácia e eficiência. O método de estudo do caso não é fácil como alguns cientistas sociais pensam, porque exige do pesquisador certos requisitos e habilidades. Na visão do Yin (2010, p. 94), "as demandas de um estudo de caso sobre o seu intelecto, seu ego e suas emoções são muito maiores do que as de qualquer outro método de pesquisa. Isso ocorre porque os procedimentos de coleta dos dados não são rotineiros", como acontece nos experimentos de laboratório.

Dada a essa complexidade, o estudo de caso necessita de um pesquisador bem instruído de alta qualidade e bem treinado para orientar a pesquisa deste gênero. Embora não existem critérios de elegibilidade das habilidades dos estudos de caso, Yin (2010) aponta as que são comumente exigidas, conforme Quadro 1.

Quadro 1. Habilidades exigidas do pesquisador de estudo de caso

\begin{tabular}{|l|l|}
\hline \multicolumn{1}{|c|}{ Habilidades } & \multicolumn{1}{c|}{ Conceitos } \\
\hline $\begin{array}{l}\text { FORMULAR } \\
\text { QUESTÕES }\end{array}$ & $\begin{array}{l}\text { Um bom pesquisador de estudo de caso deve ser capaz de } \\
\text { formular boas questões e interpretar as respostas }\end{array}$ \\
\hline SER UM BOM OUVINTE & $\begin{array}{l}\text { Um pesquisador deve ser um bom ouvinte e não ser atrapalhado } \\
\text { por suas próprias ideologias e preconceitos }\end{array}$ \\
\hline $\begin{array}{l}\text { EXERCITAR A HABILIDADE } \\
\text { E A FLEXIBILIDADE }\end{array}$ & $\begin{array}{l}\text { Um pesquisador deve ser adaptável e flexível para que situações } \\
\text { novas possam ser vistas como oportunidades, não como ameaças }\end{array}$ \\
\hline $\begin{array}{l}\text { TER UMA NOÇÃO CLARA } \\
\text { DOS ASSUNTOS EM } \\
\text { ESTUDO }\end{array}$ & $\begin{array}{l}\text { Um pesquisador deve ter noção clara dos assuntos em estudo, } \\
\text { mesmo no modo exploratório. Essa noção reduz as proporções } \\
\text { administráveis, os eventos relevantes e a informação a serem } \\
\text { buscados }\end{array}$ \\
\hline EVITAR O VIÉS & $\begin{array}{l}\text { O pesquisador deve ser imparcial sobre as noções preconcebidas, } \\
\text { incluindo as derivadas da teoria. Portanto, ela deve ser sensível e } \\
\text { responsiva à evidência contraditória }\end{array}$ \\
\hline
\end{tabular}

Fonte: Adaptado de Yin (2010) 
Na próxima secção, apresentam-se os procedimentos metodológicos.

\section{PROCEDIMENTOS METODOLÓGICOS}

O método utilizado para o levantamento dos dados desta pesquisa foi a partir da base científica Spell (Scientific Periodicals Electronic Library), tendo "Gestão Ambiental" como palavra-chave. Quanto ao método de seleção de artigos, escolhemos os classificados como estudos de casos, publicados em resumos e periódicos. As áreas de conhecimento selecionadas na base foram Administração, Economia, Engenharia, além do idioma Português.

Foi definido como período de tempo, artigos publicados entre janeiro de 2015 e dezembro de 2018. A análise dos artigos foi no mês de outubro e novembro de 2018. Para desenvolver a análise utilizamos a quantidade de 21 estudos de caso.

Para fins de análise, foram usadas as seguintes categorias: nome das revistas publicadas e quantidade de artigos, palavras-chave, e classificação Qualis/Capes por revista. Informações da revista publicada, palavras chaves, foram encontradas com facilidade na Spell, sem que fosse necessário abrir o artigo na íntegra. Quanto à classificação das revistas, utilizamos a plataforma sucupira a partir do ISSN para obter o Qualis/Capes.

\section{ANÁLISE E INTERPRETAÇÃO DOS DADOS}

Para a análise e interpretação dos dados foram utilizados um conjunto de informações organizados em categorias no programa Excel®. Os resultados são apresentados para mostrar um panorama dos estudos de casos gerados com a temática, "Gestão Ambiental" publicados na plataforma eletrônica Spell nos anos 2015, 2016, 2017 e 2018.

O Gráfico 1 mostra a primeira categoria analisada, que se refere ao número de estudos de casos e revistas publicadas na plataforma Spell de acordo com o período mencionado anteriormente.

Gráfico 1. Número de artigos e revistas publicadas

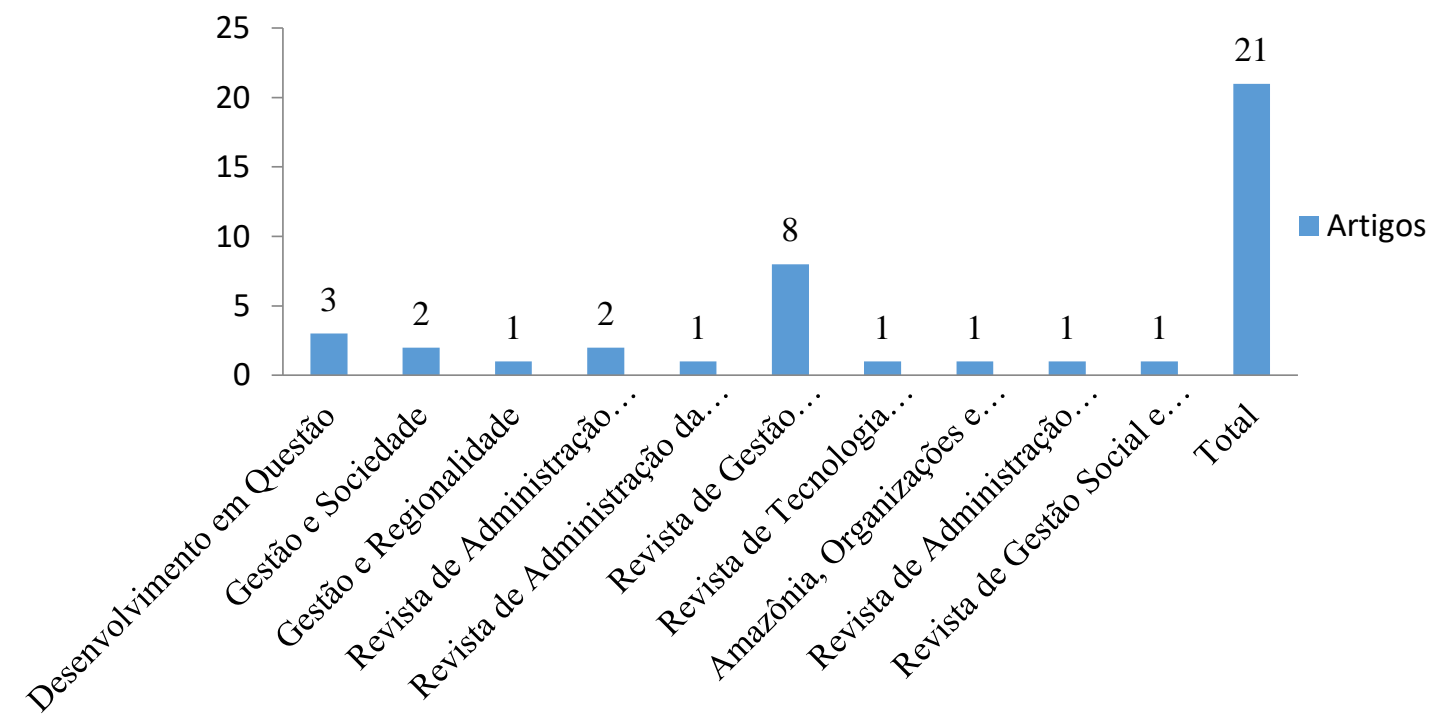

Fonte: Dados da pesquisa (2018) 
Dentre os vinte e um (21) estudos de casos analisados, oito (8) artigos foram publicados na revista de Gestão Ambiental e Sustentabilidade, três (3) artigos na revista desenvolvimento em questão, dois (2) na revista de administração da Universidade Federal de Santa Maria UFSM, dois (2) artigos na revista Gestão e Sociedade. As revistas Gestão e Regionalidade, Revista de Administração da Unimep, Revista de Tecnologia Aplicada, Amazônia, Organizações e Sustentabilidade, Revista de Administração Mackenzie e Revista de Gestão Social e Ambiental apresentaram um (1) artigo cada.

A segunda categoria analisada foi representada pelas palavras-chaves, indicadas nos resumos dos 21 estudos de caso, totalizando 226 palavras. Algumas palavras são mais frequentes, como ambiental aparece dezoito (18) vezes, gestão, treze (13) vezes, sustentabilidade e social apareceram oito (8) vezes. A Figura 1 ilustra uma nuvem das palavraschave coletas nos estudos de casos.

Figura 1. Nuvem com as palavras-chave mais utilizadas nos estudos de caso analisados.

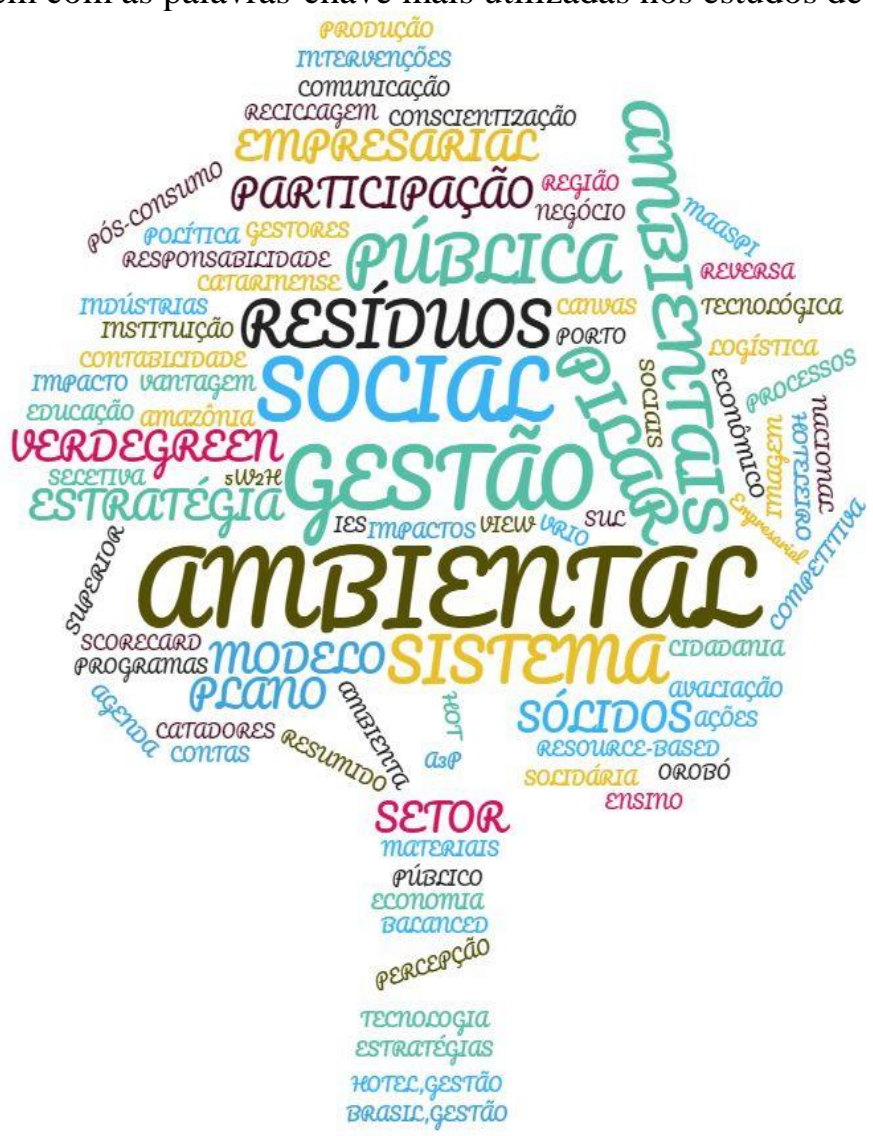

Fonte: Dados da pesquisa (2018)

Os artigos analisados quanto à abordagem de pesquisa todos são teóricos empíricos. A classificação Qualis B2, foi à classificação que mais apareceu nos estudos de caso, com dezesseis (16) artigos. Com três (3) artigos a classificação B3 e com dois (2) artigos B1. Empregando o Qualis/Capes, através do ISS de cada artigo a Quadro 1 mostra as revistas e a classificação Qualis/Capes de cada periódico. 
Quadro 1. Revista e classificação Qualis/Capes

\begin{tabular}{|c|c|c|}
\hline Revista & Qualis & Quantidade \\
\hline $\begin{array}{ll}\text { - } & \text { Revista de Administração UFSM } \\
\text { - } & \text { Amazônia, Organizações e Sustentabilidade }\end{array}$ & B1 & - 1 \\
\hline $\begin{array}{l}\text { - } \text { - } \text { Gesenvolvimento em Questão } \\
\text { - } \text { Gestão e Regionalidade } \\
\text { - } \quad \text { Revista de Administração da Unimep } \\
\text { - } \quad \text { Revista de Gestão Ambiental e } \\
\quad \text { Sustentabilidade }\end{array}$ & B2 & 16 \\
\hline $\begin{array}{l}\text { - Revista de Tecnologia Aplicada } \\
\text { - Revista de Administração Mackenzie } \\
\text { - } \quad \text { Revista de Gestão Social e Ambiental }\end{array}$ & B3 & 3 \\
\hline \multicolumn{2}{|l|}{ Total } & 21 \\
\hline
\end{tabular}

Fonte: Dados de pesquisa (2018)

\section{CONSIDERAÇÕES FINAIS}

A análise aqui apresentada teve como objetivo principal mostrar um panorama dos estudos de casos que foram publicados com o tema "Gestão Ambiental". A maioria dos estudos de casos foi publicado na revista Gestão ambiental e sustentabilidade.

O estudo analisou 226 palavras chaves, dentre elas, as que mais se destacaram foram Gestão Ambiental, Gestão Social, Sustentabilidade, Gestão de Resíduos Sólidos, Gestão Estratégica, Sistemas Ambientais. Noventa por cento (90\%) dos artigos analisados estão classificados no Qualis/Capes B2 os dez (10) restantes estão classificados como B1 ou B3.

Para futuros estudos, sugere-se a ampliação da base de dados de artigos. Este estudo foi um levantamento preliminar que possibilitará uma continuidade em estudos futuros como analisar os Estados com maior número de publicações, os principais autores, as principais temáticas e os resultados gerados pelos autores. Além disso, pode-se buscar a geração de redes de relacionamento dos autores a partir de análises envolvendo a sociometria e a bibliometria.

\section{REFERÊNCIAS}

CORAIOLA, D. M.; SANDER, J. A.; MACCALI, N.; BULGACOV, S. Estudo de Caso. In: TAKAHASHI, A. R. W. (Org.). Pesquisa Qualitativa em Administração. São Paulo: Atlas, 2013.

DE ANDRADE, S. R.; RUOFF, A. B.; PICCOLI, T.; SCHMITT, M. D.; FERREIRA, A.; XAVIER, A. C. A. O estudo de caso como método de pesquisa em enfermagem: uma revisão integrativa. Texto Contexto Enferm, v. 26, n.4, 2017.

FREITAS, W. R. S.; JABBOUR, C. Utilizando estudo de caso(s) como estratégia de pesquisa qualitativa: Boas práticas e sugestões. Estudo \& Debate, Lajeado, v. 18, n. 2, 2011.

GODOY, A. S. Introdução à Pesquisa qualitativa e suas possibilidades. Revista de administração de empresas. São Paulo, v. 35, n. 2, 1995.

GODOY, A. S. Pesquisa qualitativa. Tipos fundamentais: Revista de administração de empresas. São Paulo, v. 35, n. 3, 1995. 
KUHN, T. S. A estrutura das revoluções científicas I. Tradução Beatriz Vianna e Nelson Boeira. - 9. ed. - São Paulo: Perspectiva, 2006.

MEIRINHOS, M.; OSÓRIO, A. O estudo de caso como estratégia de investigação em educação. Inovação, Investigação em Educação. EDUSER: revista de educação, v. 2, n. 2, 2010.

SEVERINO, A. J. Metodologia do Trabalho Científico. São Paulo: Cortez Editora, 2007.

YIN, R. K. Estudo de caso: planejamento e métodos. Porto Alegre: Bookman, 2010- 248 p; 23 $\mathrm{cm}$. 